\title{
Robot-Assisted Laparoscopic Surgery for Pelvic Organ Prolapse among Peri- and Post-Menopausal Women
}

\author{
Ju Hee Kim ${ }^{1}$, Sa Ra Lee ${ }^{1}$, Eun Sil Lee ${ }^{2}$, Sung Hoon Kim¹, Hee Dong Chae \\ 'Department of Obstetrics and Gynecology, University of Ulsan College of Medicine, Asan Medical Center, Seoul, Korea, ${ }^{2}$ Department of \\ Obstetrics and Gynecology, Soonchunhyang University Seoul Hospital, Soonchunhyang University College of Medicine, Seoul, Korea
}

For patients with apical compartment pelvic organ prolapse (POP), abdominal sacrocolpopexy has been considered superior to the vaginal approach in terms of less dyspareunia and decreased risk of recurrence. Robot-assisted sacrocolpopexy (RSC) can help overcome difficulties in laparoscopic sacrocolpopexy (LSC) by facilitating deep dissection and suturing. Moreover, RSC is a safe and efficacious option for patients with POP. It has several benefits, such as its high anatomical cure rate, improvement of sexual function, reduction of perioperative complications, and low recurrence rate. In addition, it can be a safe option for elderly patients. RSC has a steep learning curve and numerous other reported advantages compared with LSC; however, insufficient data conclude that the former is universally superior, especially in cost effectiveness. Thus, further studies are needed to support the widespread adoption of robot-assisted surgery for pelvic floor reconstruction.

Key Words: Pelvic organ prolapse, Robotics, Sacrocolpopexy, Urogynecology

\section{INTRODUCTION}

Robot-assisted laparoscopic surgery has been widely performed in gynecology since the U.S. Food and Drug Administration (FDA) approved robotic surgical systems in 2005. In urogynecology, laparotomy has been the main surgical option because the surgery requires deep dissection and multiple sutures especially during sacrocolpopexy (SC) for pelvic organ prolapse (POP). However, large abdominal incisions lead to postoperative pain, increased need for analgesics, decreased postoperative ambulation, long-term hospitalization, and increased morbidity [1]. To address these issues, a minimally invasive laparoscopic technique has been developed. However, the surgery requires advanced laparoscopic surgical skills, especially for difficult suturing in deep dissected areas. Therefore, laparotomy has typically been chosen for patients with obesity, history of pelvic surgeries, and conditions such as suspected intra-abdominal adhesions or complex pathology [2] . Recent studies have shown that robotic surgery has advantages over laparoscopic surgery, such as shortening the time required to learn difficult surgical tasks, that is a steep learning curve, reducing blood loss, reducing postoperative pain, and enabling faster recovery [24]. The use of laparoscopy and robotics have improved durability of the abdominal repair while minimizing short-term morbidity associated with the open procedure [5]. A meta-analysis of robotic-assisted gynecologic surgery suggested that it has potential benefits in complex benign hysterectomy, endometrial cancer staging, myomectomy, and SC [6].

In patients with advanced-stage POP, robot-assisted sacrocolpopexy (RSC) enables full correction of the apical compartment, low mesh-related complications, and low recurrence rates [7] (Fig. 1). For patients who desire to preserve their uterus, robot-assisted sacrohysteropexy (RSH) is an ideal option, as it is associated

Received: May 17, 2020 Revised: December 13, 2020 Accepted: December 20, 2020

Address for Correspondence: Sa Ra Lee, Department of Obstetrics and Gynecology, University of Ulsan College of Medicine, Asan Medical Center, 88 Olympic-ro 43-gil, Songpa-gu, Seoul 05505, Korea

Tel: 82-2-3010-3648, E-mail: leesr@amc.seoul.kr, ORCID: https://orcid.org/0000-0002-7890-8348 


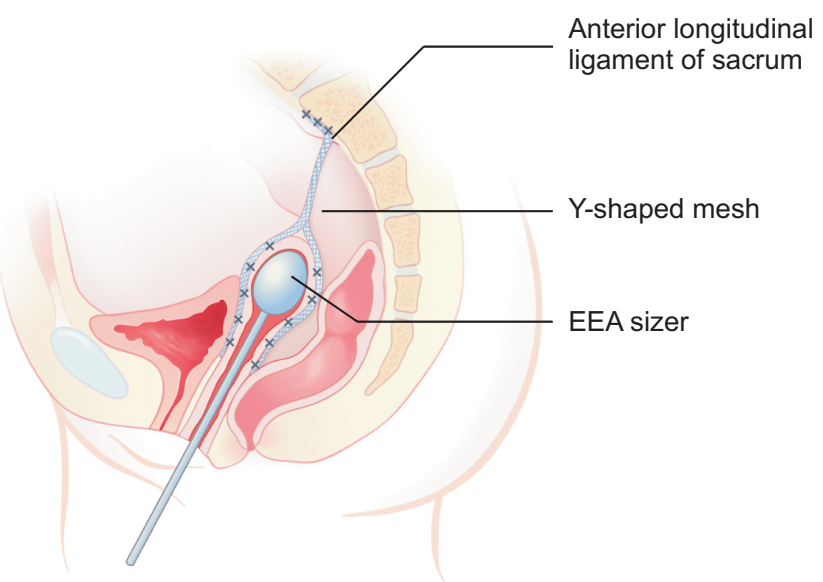

Fig. 1. Apical compartment prolapse and sacrocolpopexy. EEA: end-toend anastomosis.

with low frequency of complications and high patient satisfaction [8]. Although RSH can preserve a uterus, RSH should be performed only for women who do not have future pregnancy plan. Further, a robotic approach can provide careful dissection and reconstruction of a vesicovaginal fistula [9].

The robotic platform has developed to reduce the skin incisions by introducing the single site and single port platform. In terms of a single incision robotic surgery, in other words, robotic single port surgery (RSPS), robotic laparoendoscopic single-site using the da-Vinci $\mathrm{Xi}, \mathrm{X}$, or Si systems (Intuitive Surgical, Sunnyvale, CA, USA) or a single port robotic surgery using the daVinci SP system (Intuitive Surgical) are available. The single port platform requires only one skin incision, which improves cosmetics [10]. For various benign gynecologic surgeries, RSPS is superior to conventional laparoscopic surgery due to reduced struggling between surgical instruments; therefore, the surgeon can complete the procedure more easily and safely [11]. RSPS for SC using the Si or Xi platform has been reported to be a feasible and safe option [12-15], and recently the new SP platform has been reported to be a safe option $[11,16]$. This review article discusses robotic surgery, especially for POP.

\section{SURGICAL OUTCOMES OF RSC}

Many studies have demonstrated that robotic approaches to POP lead to successful perioperative results. van Zanten et al. [12] published a two-center prospective cohort study of robot-assisted surgery for apical prolapse. Postoperative 12-month outcomes were assessed for 305 patients who received RSC for vault prolapse $(n=188)$ and robot-assisted supracervical hysterectomy with sacrocervicopexy (RSCP; $\mathrm{n}=$ 117). Surgery was sucessful in over $90 \%$ of cases for both types of surgery ( $91 \%$ vs $99 \%$ ). Most symptomatic recurrences were located in the anterior compartment (12.1\% vs $4.8 \%)$. Symptoms of bulge improved significantly from $76.7 \%$ to $13.5 \%$ of patients. Both intraoperative and postoperative complications were few and quality of life increased after surgery [12]. Another prospective study of RSC evaluated the surgical success of RSC using lightweight Y-Mesh in 253 patients after 5 years or longer postoperatively [13]. Most patients $(89.3 \% ; 226 / 253)$ experienced objective and subjective surgical success, which was assessed as no treatment of prolapse since surgery, no mesh complications, no apical descent, and no prolapse-related symptoms. A study comparing robotic and laparoscopic sacrocolpopexy (LSC) for advanced-stage POP indicated that robotic surgery provided successful outcomes equivalent to laparoscopic surgery, with almost $100 \%$ correction of the apical compartment [7]. Over 70\% of both groups showed improved urinary symptoms, which were confirmed by uroflowmetry. Additionally, robotic surgery was associated with fewer cases of persistent prolapse than was laparoscopic surgery. These results indicate that RSC is as effective as LSC, as indicated by in pelvic organ prolapse quantification (POP-Q) of stage 3 and 4 patients [7].

\section{TOTAL OPERATIVE TIME OF RSC SURGERY}

The results of articles that compared surgery duration between robotic surgery and laparoscopic surgery have varied. Initial reports suggested that RSC was associated with longer surgery durations than was LSC. Illiano et al. [7] indicated that operation duration for RSC was longer than that for LSC (mean \pm standard deviation; $234.4 \pm 50$ minutes vs $192.75 \pm 65$ minutes), but this did not separately reflect the docking time [7]. Seror et al. [14] evaluated strictly the operation duration of RSC and LSC. The strict operation duration was defined as from port insertion to pure surgery end time, excluding the docking of the robot. The results indicated that the pure operating time of RSC was 95 minutes less than the LSC duration of 220 minutes, and demonstrated that robotic surgery was superior to laparoscopic surgery in terms of simplicity of dissection and multiple suturing capability. The duration of robotic surgery 
for POP differs depending on the surgical technique. van Zanten et al. [12] reported that the duration of RSCP was approximately 40 minutes longer than that of RSC ( $183.1 \pm 38.2$ minutes vs $145.3 \pm 29.8$ minutes $)$. However, when conducted by an experienced robotic surgeon, the difference in duration between RSC and LSC was only 13.6 minutes [14]. Additionally, the mean RSC operation duration was $122.2 \pm 22.5$ minutes, significantly less than the duration [12] and similar to the 125 minutes reported by Seror et al. [14]. First clinical experience reports of the new da Vinci SP platform indicated that the operation duration was 140.7 minutes [16]. Therefore, it appears that the surgery duration for single incision RSC, excluding the docking time, varies according to the surgeon's experience. It is likely that RSC will be faster than LSC given the faster intracorporeal suturing in robotic surgery.

\section{RSC IN ELDERLY PATIENTS}

A retrospective cohort study of the relationship between age and morbidity after urogynecologic surgery found that elderly women had a higher risk of complications compared to women under 60 years [17]. Although there are few studies of robotic surgery applied to older patients, the available studies suggest that robotic surgery is an alternative treatment option for elderly patients because it is safe and has minimal perioperative complications $[17,18]$. A study that evaluated perioperative adverse events, such as wound problems, infection, and pulmonary or cardiac problems, in patients who received RSC found no difference between those aged younger than 65 years versus those aged 65 or older (18.5\% vs $18.0 \%$ ) [18]. Robinson et al. [19] evaluated perioperative complications in women over the age of 65 years who received urogynecologic surgery. Robotic urogynecologic surgery was associated with less blood loss and fewer postoperative complications compared to vaginal approach surgery $(20.9 \%$ vs 43.3\%). There were more urinary tract infections and postoperative blood transfusions in the vaginal surgery group and the overall postoperative complications rate of the robotic surgery group was consistent with Hong (20.9\% vs $18.3 \%$ ) [19], which suggests that robotic surgery is a safe treatment option for elderly patients.

\section{RSC AND RECOVERY OF SEXUAL FUNCTION}

POP negatively affects sexual function of women be- cause of symptoms of vaginal bulging, dyspareunia, and decreased genital sensation in response to vibration and thermal stimulation compared to normal pelvic anatomy [20]. The effect of RSC on vaginal sensation was also assessed in the same cohort [21]. Although the number of patients was small $(\mathrm{n}=18)$, all demonstrated improved thermal genital sensation after RSC [21]. van Zanten et al. [22] conducted a prospective study of the sexual function of 107 patients who received RSC or robotic RSH [22]. Sexual function before and after surgery was assessed using the Pelvic Organ Prolapse/ Urinary Incontinence Sexual Questionnaire (PISQ12). The PISQ-12 results showed that, the number of women avoiding sex decreased significantly from $24 \%$ to $2 \%$ after the surgery, while the number of women who reported dyspareunia decreased from $29 \%$ to $17 \%$ [22]. A study of sexual function after LSC reported the proportion of patients who avoided sex because of bulging in the vagina decreased from $63.2 \%$ to $4.0 \%$, and proportion who reported feeling pain during intercourse decreased from $47.4 \%$ to $26.3 \%$ [23]. These results demonstrate that robotic surgery is effective for improving sexual function in women with POP.

\section{DISADVANTAGES OF RSC}

In terms of postoperative pain, Paraiso et al. [24] found that patients who received RSC reported higher pain scores and used nonsteroidal anti-inflammatory drugs during rest and normal activity within 5 weeks of surgery more frequently than did patients who received LSC. However, these results may be due to differences in the size and number of ports used for the two surgeries, with more and larger ports used in RSC: four ports (two $5 \mathrm{~mm}$ trocars, one $10 \mathrm{~mm}$ trocar, one $12 \mathrm{~mm}$ trocar) in LSC, and five ports (three $8 \mathrm{~mm}$ trocars, two $12 \mathrm{~mm}$ trocars) with an inverted $\mathrm{W}$ plan in RSC. Although there exist few reports of RSPS for pelvic floor reconstruction, a report of single-site RSC $(\mathrm{n}=25)$ indicated that only two patients required oral opioids, and no patient needed intravenous analgesics during the median two-day hospitalization [24]. Previous studies of laparoscopic single-port surgery demonstrated that reducing the number and size of ports led to reduced postoperative pain; reduced need for pain medications; reduced incision-related complications, such as infection or bleeding; and better cosmetic outcomes $[10,24]$. Therefore, it is likely that single port robotic surgery will also reduce postoperative pain and 
complications.

Regarding cost, robotic surgery is more expensive than is laparoscopic surgery. Paraiso et al. [24] published in 2011 that RSC cost approximately $\$ 2,000$ more than LSC, predominately because of differences in costs in the operating room. However, Matanes et al. [25] suggested that RSPS rather than a multiport approach could reduce the cost of surgery by reducing the use of instruments and hospitalization days. However, further studies are needed to determine the cost-effectiveness of robotic surgery $[10,24]$.

In this review, we summarized the results of research into the use of robotic surgery in urogynecology. For patients with apical compartment POP, abdominal SC has been considered the gold-standard surgical option, given its lower recurrence rate and lower incidence of dyspareunia compared to a vaginal approach. However, the introduction of RSC has the benefits of a short learning curve versus the difficulties associated with LSC, reduced morbidity, and improved recovery [2328]. RSC is beneficial in many ways, such as its high anatomical cure rate, improved sexual function outcomes, reduced perioperative complications, and low recurrence rate; it also can be a safe treatment option for elderly patients. In addition, with recent advances in RSPS, cosmetic effects have improved and procedurerelated pain has decreased [11]. Robotic laparoscopic surgery has many advantages, but there remains insufficient data to conclude that such surgery is universally the best option. More investigations are needed into aspects such as total operative time including robot docking time; postoperative long-term outcomes; and costeffectiveness. Such knowledge would contribute to the widespread adoption of robotic surgery for pelvic floor reconstruction.

\section{CONCLUSION}

RSC is a safe and efficacious option for patients with POP. Robotic surgery is particularly beneficial in urogynecology, most of whose patients are elderly women who require the shortest possible operation time and early ambulation for fast recovery.

\section{ACKNOWLEDGMENTS}

We deeply appreciated to Sun Joo Kim for medical illustration visualizing our concept.

\section{CONFLICT OF INTEREST}

No potential conflict of interest relevant to this article was reported.

\section{REFERENCES}

1. Jayakumaran J, Patel SD, Gangrade BK, Narasimhulu DM, Pandian SR, Silva C. Robotic-assisted laparoscopy in reproductive surgery: a contemporary review. J Robot Surg 2017; 11: 97-109.

2. Holloway RW, Patel SD, Ahmad S. Robotic surgery in gynecology. Scand J Surg 2009; 98: 96-109.

3. Gala RB, Margulies R, Steinberg A, Murphy M, Lukban J, Jeppson $\mathrm{P}$, et al. Systematic review of robotic surgery in gynecology: robotic techniques compared with laparoscopy and laparotomy. J Minim Invasive Gynecol 2014; 21: 353-61.

4. Lee S, Kim MR, Seong SJ, Paek J, Lee YS, Nam EJ, et al. Trends in robotic surgery in Korean gynecology. Gyne Robot Surg 2020; 1 : 50-6.

5. Coolen AWM, van Oudheusden AMJ, Mol BWJ, van Eijndhoven HWF, Roovers JWR, Bongers MY. Laparoscopic sacrocolpopexy compared with open abdominal sacrocolpopexy for vault prolapse repair: a randomised controlled trial. Int Urogynecol J 2017; 28 : 1469-79.

6. Varghese A, Doglioli M, Fader AN. Updates and controversies of robotic-assisted surgery in gynecologic surgery. Clin Obstet Gynecol 2019; 62: 733-48.

7. Illiano E, Ditonno P, Giannitsas K, De Rienzo G, Bini V, Costantini E. Robot-assisted vs laparoscopic sacrocolpopexy for high-stage pelvic organ prolapse: a prospective, randomized, single-center study. Urology 2019; 134: 116-23.

8. Li XL, Liu ZY, Zhou N, Zhu T, Yang YZ, Yao YQ. Long-term results of robotic sacral hysteropexy for pelvic organ prolapse in China Single medical center. Int J Surg 2016; 27: 128-32.

9. Sundaram BM, Kalidasan G, Hemal AK. Robotic repair of vesicovaginal fistula: case series of five patients. Urology 2006; 67: 970-3.

10. Matanes E, Lauterbach R, Boulus S, Amit A, Lowenstein L. Robotic laparoendoscopic single-site surgery in gynecology: a systematic review. Eur J Obstet Gynecol Reprod Biol 2018; 231: 1-7.

11. Shin HJ, Yoo HK, Lee JH, Lee SR, Jeong K, Moon HS. Robotic single-port surgery using the da Vinci $\mathrm{SP}^{\circledR}$ surgical system for benign gynecologic disease: a preliminary report. Taiwan J Obstet Gynecol 2020; 59: 243-7.

12. van Zanten F, Schraffordt Koops SE, O'Sullivan OE, Lenters E, Broeders I, O'Reilly BA. Robot-assisted surgery for the management of apical prolapse: a bi-centre prospective cohort study. BJOG 2019; 126: 1065-73.

13. Culligan PJ, Lewis C, Priestley J, Mushonga N. Long-term outcomes of robotic-assisted laparoscopic sacrocolpopexy using light- 
weight Y-mesh. Female Pelvic Med Reconstr Surg 2020; 26: 202-6.

14. Seror J, Yates DR, Seringe E, Vaessen C, Bitker MO, ChartierKastler E, et al. Prospective comparison of short-term functional outcomes obtained after pure laparoscopic and robot-assisted laparoscopic sacrocolpopexy. World J Urol 2012; 30: 393-8.

15. Lee SR. Robotic Single-Site ${ }^{\circledR}$ sacrocolpopexy: first report and technique using the Single-Site ${ }^{\circledR}$ wristed needle driver. Yonsei Med J 2016; 57: 1029-33.

16. Lee SR, Kim S, Chae H, Kang BM. Single-port robotic-assisted sacrocolpopexy using the SP surgical system: first clinical experience. J Minim Invasive Gynecol 2019; 26 (7 Suppl): S74.

17. Sung VW, Weitzen S, Sokol ER, Rardin CR, Myers DL. Effect of patient age on increasing morbidity and mortality following urogynecologic surgery. Am J Obstet Gynecol 2006; 194: 1411-7.

18. Hong CX, Halani PK, Gutkind N, Harvie HS, Arya LA, Andy UU. Perioperative adverse events in women over age 65 undergoing robot-assisted sacrocolpopexy. Int Urogynecol J 2020; 31: 1463-70.

19. Robinson BL, Parnell BA, Sandbulte JT, Geller EJ, Connolly A, Matthews CA. Robotic versus vaginal urogynecologic surgery: a retrospective cohort study of perioperative complications in elderly women. Female Pelvic Med Reconstr Surg 2013; 19: 230-7.

20. Gruenwald I, Mustafa S, Gartman I, Lowenstein L. Genital sensation in women with pelvic organ prolapse. Int Urogynecol J 2015; 26: 981-4.

21. Lowenstein L, Mustafa-Mikhail S, Gartman I, Gruenwald I. The effect of pelvic organ prolapse repair on vaginal sensation. Int Urogynecol J 2016; 27: 915-8.

22. van Zanten F, Brem C, Lenters E, Broeders IAMJ, Schraffordt Koops SE. Sexual function after robot-assisted prolapse surgery: a prospective study. Int Urogynecol J 2018; 29: 905-12.

23. Salamon CG, Lewis CM, Priestley J, Culligan PJ. Sexual function before and 1 year after laparoscopic sacrocolpopexy. Female Pelvic Med Reconstr Surg 2014; 20: 44-7.

24. Paraiso MF, Jelovsek JE, Frick A, Chen CC, Barber MD. Laparoscopic compared with robotic sacrocolpopexy for vaginal prolapse: a randomized controlled trial. Obstet Gynecol 2011; 118: 1005-13.

25. Matanes E, Lauterbach R, Mustafa-Mikhail S, Amit A, Wiener Z, Lowenstein L. Single port robotic assisted sacrocolpopexy: our experience with the first 25 cases. Female Pelvic Med Reconstr Surg 2017; 23: e14-8.

26. Lee IO, Yoon JW, Chung D, Yim GW, Nam EJ, Kim S, et al. A comparison of clinical and surgical outcomes between laparo-endoscopic single-site surgery and traditional multiport laparoscopic surgery for adnexal tumors. Obstet Gynecol Sci 2014; 57: 386-92.

27. Maher C, Baessler K, Glazener CM, Adams EJ, Hagen S. Surgical management of pelvic organ prolapse in women: a short version Cochrane review. Neurourol Urodyn 2008; 27: 3-12.

28. Giannini A, Russo E, Malacarne E, Cecchi E, Mannella P, Simoncini T. Role of robotic surgery on pelvic floor reconstruction. Minerva Ginecol 2019; 71: 4-17. 